\title{
Circulating brain-derived neurotrophic factor, leptin, neuropeptide $Y$, and their clinical correlates in cystic fibrosis: a cross-sectional study
}

\author{
Jan K. Nowak ${ }^{1}$, Mariusz Szczepanik ${ }^{1}$, Magdalena Trypuć ${ }^{1}$, Andrzej Pogorzelski², \\ Waldemar Bobkowski³, Marcin Grytczuk ${ }^{3}$, Alina Minarowska ${ }^{4}$, Rafał Wójciak ${ }^{5}$, Jaroslaw Walkowiak ${ }^{1}$
}

\author{
${ }^{1}$ Department of Pediatric Gastroenterology and Metabolic Diseases, Poznan University \\ of Medical Sciences, Poznan, Poland \\ ${ }^{2}$ Department of Pneumology and Cystic Fibrosis, Institute of Tuberculosis and Lung \\ Diseases, Rabka, Poland \\ ${ }^{3}$ Department of Pediatric Cardiology, Nephrology and Hypertension, Poznan University \\ of Medical Sciences, Poznan, Poland \\ ${ }^{4}$ Pediatric Pulmonology and Cystic Fibrosis Outpatient Clinic, Children's University \\ Teaching Hospital, Medical University of Bialystok, Bialystok, Poland \\ ${ }^{5}$ Department of Clinical Psychology, Poznan University of Medical Sciences, Poznan, \\ Poland
}

Submitted: 31 August 2017

Accepted: 25 October 2017

Arch Med Sci 2020; 16 (5): 1049-1056

DOI: https://doi.org/10.5114/aoms.2018.75322

Copyright (c) 2018 Termedia \& Banach

\section{Abstract}

Introduction: Cystic fibrosis (CF) involves chronic inflammation and decreased pulmonary function, which increase caloric demand. Yet, sufficient energy provision is hindered by reduced appetite and fat malabsorption. Brain-derived neurotrophic factor (BDNF), leptin, and neuropeptide Y (NPY) belong to energy balance-regulating factors. We aimed to assess their concentrations in CF patients in order to search for potential clinical correlates. Material and methods: This was an exploratory, cross-sectional study. $\mathrm{Pa}$ tients' weight and height $Z$-scores, forced expiratory volume in $1 \mathrm{~s}(\mathrm{FEV} \%)$, exocrine pancreatic status (fecal elastase-1), genotypes, and other characteristics were assessed. Serum concentrations of BDNF, leptin, NPY, IL-6, and TNF- $\alpha$ were measured using ELISA.

Results: The study enrolled 56 patients, of whom 29 (52\%) were female and $17(30 \%)$ were younger than 16 years. Median ( $1^{\text {st }}-3^{\text {rd }}$ quartile) mass Z-score was -0.85 (-1.56-(-0.36)); median FEV was 70.5\% (45.0-89.5); 48 $(86 \%)$ patients had exocrine pancreatic insufficiency and $8(14 \%)$ diabetes. Overall, median concentrations were: BDNF: $33.91 \mathrm{ng} / \mathrm{ml}$ (26.40-40.43), leptin: $12.05 \mathrm{ng} / \mathrm{ml}$ (8.93-17.77), NPY: $2.86 \mathrm{ng} / \mathrm{ml}(1.75-4.42)$. None of these factors correlated with mass Z-score, FEV $\%$, IL- 6 or TNF- $\alpha$. Leptin and NPY correlated negatively $\left(\rho=-0.62, p=3 \times 10^{-7}\right)$; BDNF/NPY ratio was associated with leptin $\left(\rho=0.54, p=2 \times 10^{-5}\right)$, BDNF/leptin ratio correlated with NPY $\left(\rho=0.60, p=1 \times 10^{-6}\right)$. In a multivariable regression analysis NPY was weakly, but independently, associated with $\mathrm{FEV}_{1} \%$, and leptin with age.

Conclusions: BDNF and leptin were not associated with weight Z-score or FEV $\%$. Serum NPY concentrations seemed to be lower in CF patients with reduced pulmonary function independently of malnutrition and inflammation.

Key words: appetite, cachexia, malnutrition, inflammation, respiratory insufficiency.

\section{Corresponding author:} Prof. Jaroslaw Walkowiak $\mathrm{MD}, \mathrm{PhD}$

Department of Pediatric Gastroenterology and Metabolic Diseases Poznan University of Medical Sciences 27/33 Szpitalna St 60-572 Poznan, Poland Phone: +48 618491432 E-mail: jarwalk@ump.edu.pl 


\section{Introduction}

Cystic fibrosis (CF; OMIM \#219700) is a genetic disease caused by a dysfunction of the cystic fibrosis (CF) transmembrane conductance regulator (CFTR), which impairs chloride ion transport across cellular membranes. A defect in CFTR production, processing, regulation, splicing, or stability- and conductance-preserving mechanisms ultimately results in the production of abnormally viscous mucus throughout the body. This in turn provides ample nourishment to the pathogenic bacteria colonizing the respiratory tract and blocks the pancreatic ducts, in most of the patients leading to auto-digestion of the organ and exocrine pancreatic insufficiency. The ensuing recurrent infections of the lungs and the diminishing pulmonary capacity require a supply of energy larger by 10 $100 \%$ compared with the norm for the age. This need, however, is difficult to satisfy because of fat malabsorption. Thus, an atypical combination of orexigenic and anorexigenic influence exists in CF patients, and unfortunately the too-frequent outcome is insufficient feeding [1]. Understanding the physiology behind these issues is of great importance for preserving adequate nutritional status, which in CF clearly improves survival.

This study focuses on three feeding-regulating factors, of which the first is brain-derived neurotrophic factor (BDNF) [2]. BDNF is a protein expressed predominantly in the brain, but is also found in the reproductive systems (both male and female), endocrine tissues, and the lungs. It plays a role in neural development and neuroplasticity, and as such is proposed to be involved in psychiatric disorders, including depression and addictions. Genetic diseases caused by BDNF haploinsufficiency provide important clues to its functions. The WAGR syndrome with BDNF truncation and other BDNF-affecting deletions within chromosome 11 are known to cause overeating and obesity [3]. The anorexigenic function of BDNF is mediated by its receptor, tropomyosin-related kinase B (TrkB), which activates pathways involving phosphoinositide 3-kinase (PI3K) mitogen-activated protein kinases (MAPK), protein kinase $B(A k T)$, and mechanistic target of rapamycin (mTOR). There is also population-scale evidence linking BDNF to body weight regulation [4]. Furthermore, intraventricular injections of BDNF decreased appetite in rats. As a consequence, BDNF is considered one of the pivotal molecules in energy balance regulation. Acute exercise is known to increase BDNF concentrations in the peripheral blood of healthy adults, but may be dependent on the duration of physical activity and gender [5].

Leptin is a protein hormone produced by white adipocytes, which is a ligand of the leptin receptor, expressed in a wide range of tissues. It is a crucial mediator of satiety [6] and has effects opposite to those of ghrelin. Reduced sensitivity to leptin may be associated with obesity and lead to hyperleptinemia. Exceedingly rare mutations in the LEP gene - which encodes leptin - are associated with hyperphagia. Leptin levels readily change in response to a broad spectrum of stimuli, including disease, chronic physical exertion (a reduction in leptin), and other sources of stress. Of interest, leptin-deficient obese mice are known to express less CFTR in the intestine [7]. In CF, leptin levels were shown to be lower [8, 9], similar [10, 11], higher [12, 13], or higher when corrected for age and body mass [14] than in healthy volunteers, and to not differ depending on disease severity [15].

Neuropeptide Y (NPY) is a polypeptide beginning and ending with a tyrosine $(Y)$ residue, which is secreted in the central and sympathetic nervous systems and by the endocrine tissues of the gut. Four subtypes of the NPY receptor are known to display considerable effects in humans: activation of receptors Y1 and Y5 stimulates hunger [16], Y2 may be associated with satiety and play a role in autocrine regulation of NPY secretion, and Y4 is expressed in small quantities in the hypothalamus. Also in this regulatory region of the brain, NPY opposes the effects of leptin. Obesity is associated with a paradoxical elevation of NPY, which might be due to hyperleptinemia. In animal models, stress was shown to increase NPY and promote abdominal adipogenesis.

The aims of this exploratory study were to: (1) describe serum concentrations of BDNF, leptin, and NPY in patients with CF, (2) search for potential correlations between these factors, their ratios, and the clinical characteristics of CF.

\section{Material and methods}

The subjects were recruited in CF centers in Poznan and Rabka (Poland) among patients who regularly visit their physician. The inclusion criteria were CF and age of at least 7 years. The exclusion criterion was disease exacerbation necessitating intravenous antibiotics.

Body mass and height were measured and subsequently Z-scores and body mass index (BMI) were determined. Mass Z-score was used in order to evaluate nutritional status. The following information was obtained from the medical records: forced expiratory volume in $1 \mathrm{~s}\left(\mathrm{FEV}_{1} \%\right)$ as assessed in the last 3 months, exocrine pancreatic insufficiency (fecal elastase-1 concentration $<200 \mu \mathrm{g} / \mathrm{g}$ ) [17], and a diagnosis of diabetes.

Serum concentrations of BDNF, leptin, NPY, interleukin-6 (IL-6), and tumor necrosis factor $\alpha$ (TNF- $\alpha$ ) were measured using enzyme-linked immunosorbent assays (ELISA) according to the manufacturers' protocols (NPY, leptin, IL-6, TNF- $\alpha$ : Phoenix Pharmaceuticals Inc., Burlingame, USA; BDNF: Millipore Merck, Warsaw, Poland). Intra-as- 
say and inter-assay coefficients of variability were verified as meeting manufacturers' specifications (ELISAs from Phoenix - intra-assay variation 5-10\%, inter-assay variation < 15\%; Millipore - intra-assay variation $3.7 \%$, inter-assay variation $8.5 \%$ ).

The study respected the rules set by the Declaration of Helsinki and was approved by the Bioethical Committee at Poznan University of Medical Sciences, Poznan, Poland (250/10). The participants who were at least 16 years old gave informed written consent for their inclusion in the study; such consent was also provided by parents of all the enrolled underage participants.

\section{Statistical analysis}

Statistical analyses were performed in the Statistica 12.0 software environment (StatSoft Inc., Tulsa, USA). The data are presented as medians ( $1^{\text {st }}-3^{\text {rd }}$ quartile) unless specified otherwise. The Mann-Whitney $U$ test was used to compare differences in the distribution of parameter values in subgroups (two-sided $p$ ). Boolean parameters were compared between groups with Fisher's test (two-sided $p$ ). Spearman's rank-order correlation was calculated where appropriate. To compensate for confounding and identify potential associations between the factors and clinical character- istics, multivariable regression models were built using the forward stepwise method with sigma-restricted parametrization. The following variables were included: age, sex, body mass Z-score, $\mathrm{FEV}_{1} \%$, exocrine pancreatic insufficiency (all the patients with diabetes were positive and therefore diabetes was not included in the model), and IL-6. BoxPlotR was used to generate the violin plot [18].

The research was exploratory. The $\alpha$ level was set at 0.05 . Power calculations were performed using G*Power 3.1.9.2 [19]. Assuming the power of the test 0.80 , a sample size of $\geq 44$ is sufficient to detect moderate and strong correlations ( $\rho \geq 0.4$; two-tailed $p$-value; the calculation is identical as for Pearson's $r$ coefficient); $n \geq 17$ is sufficient to detect stronger correlations $(\rho \geq 0.6)$. With the power of the test 0.80 and two subsamples $\geq 17$, it is possible to detect a difference in values of a variable equaling its standard deviation. Given the above power assumptions, six tested predictors, and a target $R^{2}$ of 0.3 , a linear multivariable regression requires a sample size $\geq 39$. Additionally, two subgroups were compared: subjects at least 16 years old vs. younger subjects, since such a division between physiologically mature and immature populations certainly requires attention.

Table I. Characteristics of the investigated CF patients overall and in two age subgroups

\begin{tabular}{|lccc|}
\hline Parameter & All & Age $\geq 16$ years & Age $<16$ years \\
\hline$N$ & 56 & 39 & 17 \\
\hline Age [years] & $17.5(14.5-25.0)$ & $22.0(17.0-29.0)$ & $12.0(9.0-14.0)$ \\
\hline Sex: female & $51.8 \%(n=29)$ & $46.1 \%(n=18)$ & $64.7 \%(n=11)$ \\
\hline Genotype: & & & \\
\hline p.[Phe508del];Phe508del] & $44.6 \%(n=25)$ & $48.7 \%(n=19)$ & $35.3 \%(n=6)$ \\
\hline p.[Phe508del];other & $41.1 \%(n=23)$ & $33.3 \%(n=13)$ & $58.8 \%(n=10)$ \\
\hline Other or unknown & $14.3 \%(n=8)$ & $17.9(n=7)$ & $5.9 \%(n=1)$ \\
\hline Mass Z-score & $-0.85(-1.56-(-0.36))$ & $-0.81(-1.59-(-0.25))$ & $-1.19(-1.60-(-0.51))$ \\
\hline Mass [kg] & $48.4(29.7-56.2)$ & $54.0(46.0-58.0)$ & $27.2(22.0-29.0)$ \\
\hline Height Z-score & $-1.02(-1.88-(-0.19))$ & $-0.93(-1.79-(-0.40))$ & $-1.21(-1.96-(-0.02))$ \\
\hline Height [cm] & $160(140-166)$ & $163(156-170)$ & $135(124-137)$ \\
\hline BMI [kg/m²] & $18.4(15.7-20.7)$ & $19.6(17.8-22.0)$ & $15.3(14.5-15.9)$ \\
\hline BMI [percentile] & $21(7-58)$ & $28(10-69)$ & $12(1-26)$ \\
\hline FEV $\%$ & $70.5(45.0-89.5)$ & $67.0(45.0-86.0)$ & $80.0(54.0-96.0)$ \\
\hline Exocrine pancreatic insufficiency & $85.7 \%(n=48)$ & $84.6 \%(n=33)$ & $88.2 \%(n=15)$ \\
\hline Diabetes & $14.3 \%(n=8)$ & $17.9 \%(n=7)$ & $5.9 \%(n=1)$ \\
\hline TNF- $\alpha[p g / m l]$ & $19.1(17.4-23.2)$ & $19.0(17.8-24.1)$ & $19.1(16.9-22.8)$ \\
\hline IL-6 [pg/ml] & $4.56(3.05-5.91)$ & $4.34(3.02-6.07)$ & $4.98(4.09-5.67)$ \\
\hline
\end{tabular}

Medians ( $1^{\text {st }}-3^{\text {rd }}$ quartiles) are reported. BMI - body mass index, FEV ${ }_{1} \%$ - forced expiratory volume in one second (\%), IL-6 - interleukin 6 , TNF- $\alpha$-tumor necrosis factor $\alpha$. 


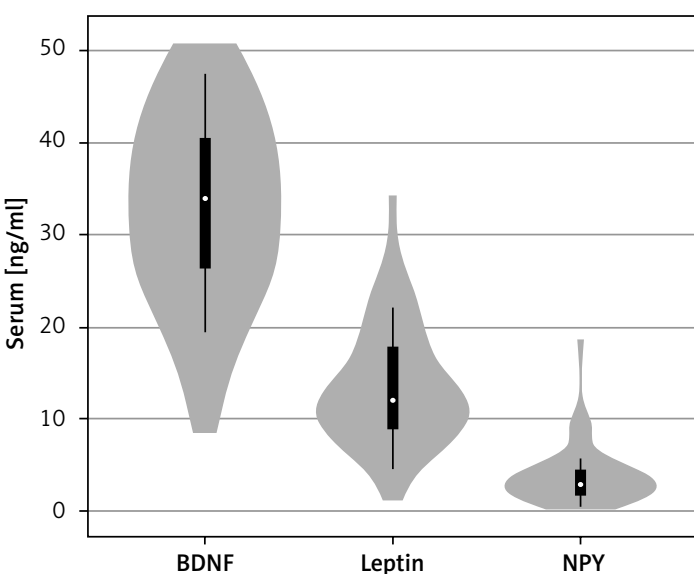

Figure 1. Violin plot of serum concentrations of brain-derived neurotrophic factor (BDNF), leptin, and neuropeptide $Y$ (NPY) in CF patients. White points correspond to medians; boxes represent $1^{\text {st }}$ $3^{\text {rd }}$ quartiles; whiskers extend to the extreme values, which still lie within $1.5 \times$ interquartile range from the $1^{\text {st }}$ and the $3^{\text {rd }}$ quartile (Tukey's method)

\section{Results}

\section{Patient characteristics}

Fifty-six CF patients were recruited for the study (Table I). Mass Z-scores, height Z-scores, and $\mathrm{FEV}_{1} \%$ were similar in patients at least 16 years old and in the younger subgroup ( $p=$ $0.791, p=0.972$, and $p=0.214$, respectively); no differences were also found in proportions of exocrine pancreatic insufficiency $(p=1.0)$ and diabetes $(p=0.412)$. Mass, height, and $\mathrm{BMI}$ were lower in subjects $<16$ years $\left(p=3 \times 10^{-10}, p=2 \times 10^{-8}\right.$, $p=8 \times 10^{-8}$ ). Patients' CFTR genotypes are listed in Table II.

\section{Serum concentrations of BDNF, leptin, and} NPY

Serum concentrations of BDNF, leptin, and NPY are presented in Table III; their distribution is shown in Figure 1.

Table II. Patients' CFTR genotypes. Both legacy and Human Genome Variation Society (HGVS) names are given

\begin{tabular}{|c|c|c|}
\hline Legacy name & HGVS name & $N$ \\
\hline F508del/F508del & p.[Phe508del];[Phe508del] & 25 \\
\hline F508del/- & p.Phe508del & 7 \\
\hline F508del/2143delT & p.[Phe508del];[Leu761X] & 4 \\
\hline Unknown & Unknown & 4 \\
\hline F508del/CFTRdele2,3 & p.Phe508del;c.54-5940_273+10250del21kb & 2 \\
\hline F508del/R553X & p.[Phe508del];[Arg553X] & 2 \\
\hline $3272-26 A G /-$ & c. $3140-26 A>G$ & 1 \\
\hline 3659delC/- & p.Lys1177SerfsX15 & 1 \\
\hline F508del/1717-1GA & p.Phe508del;c.1585-1G>A & 1 \\
\hline F508del/2183AAG & p.[Phe508del];[Lys684SerfsX38] & 1 \\
\hline F508del/3849+10kbC->T & p.Phe508del;c.3717+12191C>T & 1 \\
\hline F508del/621+1GT & p.Phe508del;c.489+1G>T & 1 \\
\hline F508del/c.-674T>C & p.Phe508del;c.-674T>C & 1 \\
\hline F508del/G1244R & p.[Phe508del];[Gly1244Arg] & 1 \\
\hline F508del/G27V & p.[Phe508del];[Gly27Val] & 1 \\
\hline F508del/R668C/3849+10kbC->T & p.[Phe508del;Arg668Cys];c.3717+12191C>T & 1 \\
\hline G542X/- & p.Gly542X & 1 \\
\hline T581l/2721del11 & p.[Ile864SerfsX28];[Thr581Ile] & 1 \\
\hline
\end{tabular}

Table III. Serum concentrations of brain-derived neurotrophic factor (BDNF), leptin, and neuropeptide Y (NPY) in CF patients: overall and depending on age

\begin{tabular}{|lcccc|}
\hline Parameter & All, $n=56$ & Age $\geq 16$ years, $n=39$ & Age $<16$ years, $n=17$ & $P$-value \\
\hline BDNF $[\mathrm{ng} / \mathrm{ml}]$ & $33.91(26.40-40.43)$ & $33.28(23.25-40.43)$ & $35.93(36.65-40.43)$ & NS. 0.659 \\
& $32.80 \pm 10.41$ & $32.39 \pm 11.00$ & $33.74 \pm 9.15$ & 0.001 \\
\hline Leptin $[\mathrm{ng} / \mathrm{ml}]$ & $12.05(8.93-17.77)$ & $13.01(10.07-18.97)$ & $8.78-12.01)$ & $9.60 \pm 4.70$ \\
& $13.46 \pm 6.33$ & $15.14 \pm 6.25$ & $3.69(2.62-8.71)$ & 0.034 \\
\hline NPY $[\mathrm{ng} / \mathrm{ml}]$ & $2.86(1.75-4.42)$ & $2.72(1.53-4.15)$ & $5.41 \pm 4.40$ & \\
\hline
\end{tabular}

The values were compared between the two subgroups. Medians ( $1^{\text {st }}-3^{\text {rd }}$ quartiles) and means \pm SD are presented. 
Table IV. A correlation matrix of brain-derived neurotrophic factor (BDNF), leptin, neuropeptide Y (NPY), and their ratios with main continuous parameters describing the cystic fibrosis group. Spearman's $\rho$ and $p$-value are reported

\begin{tabular}{|lcccccc|}
\hline Parameter & BDNF & Leptin & NPY & BDNF/leptin & BDNF/NPY & Leptin/NPY \\
\hline Age & -0.12 & -0.28 & 0.43 & -0.43 & 0.23 & 0.36 \\
& NS. 0.368 & 0.035 & 0.001 & 0.001 & NS. 0.086 & 0.005 \\
\hline Mass & 0.02 & 0.23 & 0.05 & -0.15 & -0.10 & 0.05 \\
Z-score & NS. 0.857 & NS. 0.09 & NS. 0.729 & NS. 0.287 & NS. 0.469 & NS. 0.720 \\
\hline FEV $\%$ r & 0.15 & -0.10 & 0.25 & 0.21 & -0.19 & -0.19 \\
& NS. 0.278 & NS. 0.466 & NS. 0.065 & NS. 0.116 & NS. 0.159 & NS. 0.156 \\
\hline IL-6 & 0.08 & 0.11 & -0.05 & -0.02 & 0.08 & 0.06 \\
& NS. 0.578 & NS. 0.414 & NS. 0.730 & NS. 0.909 & NS. 0.545 & NS. 0.648 \\
\hline TNF- $\alpha$ & -0.11 & 0.21 & -0.23 & -0.18 & 0.19 & 0.23 \\
& NS. 0.398 & NS. 0.123 & NS. 0.088 & NS. 0.196 & NS. 0.156 & NS. 0.086 \\
\hline
\end{tabular}

FEV $\%$ - forced expiratory volume in $1 \mathrm{~s}(\%)$, IL-6-interleukin 6, TNF- $\alpha$-tumor necrosis factor $\alpha$.

\section{BDNF, leptin, and NPY vs. clinical characteristics}

None of the three factors correlated with mass Z-score or $\mathrm{FEV}_{1} \%$ (Table IV). The analysis of scatterplots did not reveal potential non-linear trends. BDNF, leptin, and NPY did not differ depending on sex (all $p>0.25$ ) or exocrine pancreatic function status (all $p>0.7)$. When diabetic $(n=8)$ and non-diabetic $(n=40)$ exocrine pancreatic-insufficient patients were compared, a slight difference in serum leptin concentrations was found (17.14 $\mathrm{ng} / \mathrm{ml}$ (12.39-20.04) vs. $11.42 \mathrm{ng} / \mathrm{ml}$ (8.89-15.33), respectively, $p=0.043$; other $p>0.23)$. BDNF/ leptin, BDNF/NPY, and leptin/NPY ratios were similar regardless of $\operatorname{sex}(p>0.1)$ and exocrine pancreatic insufficiency (all $p>0.5$ ). In diabetic pancreatic-insufficient patients the BDNF/leptin ratio was lower (1.46 (1.10-2.65) vs. 3.11 (1.854.16), $p=0.020$; of note, 7 out of 8 diabetics were adults), whereas the other two ratios did not differ in this comparison.

Overall, BDNF did not correlate with leptin or NPY, but leptin was associated with NPY $(\rho=-0.62$, $\left.p=3 \times 10^{-7}\right)$. BDNF/NPY ratio was linked to leptin $\left(\rho=0.54, p=2 \times 10^{-5}\right)$, BDNF/leptin ratio correlated with NPY $\left(\rho=0.60, p=1 \times 10^{-6}\right)$, but leptin/ NPY was not associated with BDNF. Very similar relationships were observed in the subgroup aged $\geq 16$ years and also in children < 16 years; in the latter only BDNF/NPY-leptin correlation did not occur.

The multivariable regression model was not valid for BDNF (nor for BDNF/NPY or leptin/NPY ratios). NPY weakly, but independently, associated with $\mathrm{FEV}_{1} \%$ and leptin correlated with age (for both: $\beta=0.36,95 \% \mathrm{Cl}$ : 0.10-0.61; whole model $\left.R^{2}=0.11, p=0.007\right)$. BDNF/leptin ratio was found to depend on $\mathrm{FEV}_{1} \%(\beta=0.28,95 \% \mathrm{Cl}$ : 0.02-0.55; whole model $\left.R^{2}=0.08, p=0.03\right)$, although this observation seemed to rely on one outlying value, which we found no good reason to exclude (after exclusion: an effect only for age, $\beta=-0.37,95 \% \mathrm{Cl}$ :
-0.63-(-0.12); whole model $\left.R^{2}=0.14, p=0.005\right)$. The analysis of residuals in the remaining cases did not reveal heteroscedasticity, nonlinearity, or other issues.

\section{Discussion}

This is the first study to measure BDNF and NPY in CF patients, and to investigate their potential clinical correlates. The results suggest that poorer respiratory function might be associated with lower NPY and in this way contribute to insufficient food intake. This research did not identify other relations between characteristics of the disease and BDNF, leptin, or NPY.

Despite the evidence for appetite dysregulation in CF, our knowledge of the underlying mechanisms is insufficient. In 2008, Cohen et al. found ghrelin to be elevated and leptin to be decreased in CF patients with severe disease ( $\mathrm{FEV}_{1} 27 \pm 7 \%$ ). Ghrelin correlated with $\mathrm{FEV}_{1} \%$ (an independent predictor), TNF- $\alpha$, and IL-6; leptin associated with $\mathrm{BMI}, \mathrm{FEV}_{1} \%$, and TNF- $\alpha$ [9]. The authors concluded that the observed changes in ghrelin and leptin concentrations were due to weight loss rather than CF itself. The same team also reported that leukocytic expression of the ghrelin receptor was reduced in cachectic CF subjects and temporarily decreased during exacerbations in CF patients with normal BMI [20]. Another study, which was conducted in 30 children with CF, revealed higher plasma ghrelin levels than in healthy subjects, although there were no results of multivariable regression to compensate for confounding [21].

In 2013, Cohen et al. observed increased plasma nesfatin- 1 concentrations in CF patients with more advanced disease and lower fat mass; this was surprising because nesfatin-1 is an anorexigenic factor. This effect was not found to be related to IL-6 and TNF- $\alpha$ [22].

Notably, CF could affect the brain itself: after CFTR mRNA was found in the hypothalamus - the very center of energy balance regulation feedback 
loops - its expression and the presence of the protein product were confirmed in the neurons throughout the brain [23] and in the ganglia of the gut [24], raising the possibility that CF affects the development and the function of the brain, as well as the gut-brain axis.

The problem of reduced food intake in CF has led to a number of trials of appetite stimulants, some of which gave positive results [25]. Nevertheless, their use - bringing about undesirable effects - is not recommended in all patients [1]. One exception could be zinc supplementation in case of deficiency.

This study did not identify strong correlations of circulating BDNF, leptin, and NPY with clinical parameters. Serum BDNF in CF patients was similar to values reported in healthy persons previously (means or medians in the range 18-49 ng/ $\mathrm{ml}$ [26-28]. Three states need to be considered with regard to BDNF: malnutrition, inflammation, and physical exertion. Underweight patients with anorexia nervosa tend to have lower circulating BDNF, which should stimulate appetite [29]. The relation between immune response and circulating BDNF is unclear; following surgery serum and plasma BDNF levels moderately correlated with transforming growth factor- $\beta 1$ (which decreased) and TNF- $\alpha$ (which increased), respectively, suggesting that serum BDNF reflects platelet activation [30]. In depression, an increase in TNF- $\alpha$ over the long term is accompanied by a decrease in serum BDNF [31]. As already mentioned, BDNF levels are increased in exercise [5] - although in some children the opposite might be true [32]. In summary, the influences of malnutrition, inflammation, and increased physical effort could level off in CF patients.

Circulating leptin concentrations are known to reflect total body fat and to be higher in females than in males. In our patients, serum leptin in men and women did not differ, and its levels were comparable with those of control subjects in previous studies $[33,34]$. Leptin was lower in children, as could be expected. We did not observe the previously reported positive association with IL-6 [35]. The finding that leptin levels did not correlate with body Z-scores in our CF patients occupies the middle ground between the aforementioned conflicting results.

The data on circulating NPY are scarce: in a study by Huang et al. plasma NPY was lower in patients with primary insomnia than in healthy subjects (5.11 \pm 2.87 vs. $7.01 \pm 3.44 \mathrm{ng} / \mathrm{ml}$ ) [36]; serum NPY in healthy children was $3 \mathrm{ng} / \mathrm{ml}$ [37]. NPY levels in the blood described by diverse groups vary greatly, probably depending on the antibody used (from 0.2 to over $30 \mathrm{ng} / \mathrm{ml}$ ). NPY is increased by prolonged exercise [38] but is low in critically ill patients [39]. The possibility that NPY is reduced in CF, especially with significant respiratory insufficiency, warrants further research.

Our study did not identify the majority of the hypothesized relations between the energy balance-regulating factors and the clinical parameters. We would like to propose three explanations. In the first place, it might be that none of the three factors is responsible for the reduced appetite in CF. Secondly, energy balance-regulating mechanisms in patients with CF could be able to adapt to a state of chronic inflammation through desensitization. And third, the effects may be smaller than we expected: in this case the study would be underpowered.

We explored the correlations of BDNF, leptin, and NPY, and also searched for predictors of their ratios, because they are known to interact in the regulation of feeding. BDNF and NPY negatively control each other and higher leptin concentration is associated with lower NPY. One might ask whether the extent to which they influence each other changes depending on the characteristics of CF. We did not detect such relationships, but we did find evidence for a negative correlation between leptin and NPY and a positive association between BDNF/leptin ratio and NPY. These relations were moderate to strong. This not only hints at their potential value in future energy balance research, but also exposes the need to develop comprehensive profiling of circulating appetite-regulating molecules, then build a model of their network of interactions, and also study the impact of disease on the state of this network.

The main limitations of this exploratory study include: the fact that inferences about central energy balance are attempted by measuring markers in the peripheral blood, the lack of a healthy and age-matched control group, the cross-sectional design with only one sample taken from each patient, the lack of measures of fat mass, appetite, sweat chloride, and Pseudomonas aeruginosa colonization. It is well known that the concentrations of food intake-regulating hormones are subject to considerable diurnal variation, whereas we limited our investigation to one sample only. Forward stepwise multivariable regression, which often indicates misleadingly large effects, was used to compensate for confounding, and the results that it yielded should be treated with caution. The characteristics of the included population also warrant a comment: the recruited patients visit the clinic frequently, in many cases because of more severe CF expression. The sample is therefore moderately biased towards lower $Z$-scores and $\mathrm{FEV}_{1} \%$ values. Moreover, it includes children, because median survival is < 35 years. Finally, ELISA measurements have their own constraints resulting not only from 
the choice of the antibodies, but also from other aspects, such as the conditions of assessment; as a consequence, the obtained results can seldom be reliably compared across separate research projects.

In conclusion, in CF patients BDNF and leptin did not correlate with weight Z-score or $\mathrm{FEV}_{1} \%$. Serum NPY concentrations, however, seemed to be lower in CF patients with reduced pulmonary function independently of malnutrition and inflammation. None of the three investigated factors was associated with exocrine pancreatic insufficiency.

\section{Acknowledgments}

The authors would like to thank the patients for their participation in the study.

\section{Conflict of interest}

The authors declare no conflict of interest.

\section{References}

1. Turck D, Braegger CP, Colombo C, et al. ESPEN-ESPGHANECFS guidelines on nutrition care for infants, children, and adults with cystic fibrosis. Clin Nutr 2016; 35: 557-77.

2. Bathina S, Das UN. Brain-derived neurotrophic factor and its clinical implications. Arch Med Sci 2015; 11: 1164-78.

3. Han JC, Liu QR, Jones $M$, et al. Brain-derived neurotrophic factor and obesity in the WAGR syndrome. N Engl J Med 2008; 359: 918-27.

4. Speliotes EK, Willer CJ, Berndt SI, et al. Association analyses of 249,796 individuals reveal 18 new loci associated with body mass index. Nat Genet 2010; 42: 937-48.

5. Dinoff A, Herrmann N, Swardfager W, Lanctôt KL. The effect of acute exercise on blood concentrations of brain-derived neurotrophic factor in healthy adults: a meta-analysis. Eur J Neurosci 2017; 46: 1635-46.

6. Rosenbaum M, Leibel RL. 20 years of leptin: role of leptin in energy homeostasis in humans. J Endocrinol 2014; 223: T83-96.

7. Leung L, Kang J, Rayyan E, et al. Decreased basal chloride secretion and altered cystic fibrosis transmembrane conductance regulatory protein, Villin, GLUT5 protein expression in jejunum from leptin-deficient mice. Diabetes Metab 2014; 7: 321-30.

8. Boguszewski MCS, Kamoi TO, Bento Radominski R, et al. Insulin-like growth factor-1, leptin, body composition, and clinical status interactions in children with cystic fibrosis. Horm Res 2007; 67: 250-6.

9. Cohen RI, Tsang D, Koenig S, Wilson D, McCloskey T, Chandra S. Plasma ghrelin and leptin in adult cystic fibrosis patients. J Cyst Fibros 2008; 7: 398-402.

10. Arumugam R, LeBlanc A, Seilheimer DK, Hardin DS. Serum leptin and IGF-I levels in cystic fibrosis. Endocr Res 1998; 24: 247-57.

11. Ziai S, Belson L, Malet A, et al. The association between leptin and insulin levels in adults with cystic fibrosis. Diabetes Metab 2012; 38: 34-9.

12. Stylianou C, Galli-Tsinopoulou A, Koliakos G, Fotoulaki M, Nousia-Arvanitakis S. Ghrelin and leptin levels in young adults with cystic fibrosis: relationship with body fat. J Cyst Fibros 2007; 6: 293-6.

13. Monajemzadeh M, Ashtiani MTH, Sadrian E, et al. Variation in plasma leptin levels in young Iranian children with cystic fibrosis. Arch Med Sci 2013; 9: 883-7.

14. Ahme ML, Ong KK, Thomson AH, Dunger DB. Reduced gains in fat and fat-free mass, and elevated leptin levels in children and adolescents with cystic fibrosis. Acta Paediatr 2004; 93: 1185-91.

15. Schmitt-Grohé $S$, Hippe $V$, lgel $M$, et al. Serum leptin and cytokines in whole blood in relation to clinical and nutritional status in cystic fibrosis. J Pediatr Gastroenterol Nutr 2006; 43: 228-33.

16. Holzer P, Reichmann F, Farzi A. Neuropeptide Y, peptide $Y Y$ and pancreatic polypeptide in the gut-brain axis. Neuropeptides 2012; 46: 261-74.

17. Walkowiak J. Assessment of maldigestion in cystic fibrosis. J Pediatr 2004; 145: 285-7.

18. Spitzer M, Wildenhain J, Rappsilber J, Tyers M. BoxPlotR: a web tool for generation of box plots. Nat Methods 2014; 11: 121-2.

19. Faul F, Erdfelder E, Buchner A, Lang AG. Statistical power analyses using G*Power 3.1: tests for correlation and regression analyses. Behav Res Methods 2009; 41: 1149-60.

20. Cohen RI, Chandra S, Koenig S, Tsang D, Wilson D, MCCloskey T. Ghrelin receptor expression in lymphocytes isolated from adult cystic fibrosis patients. Respir Int Rev Thorac Dis 2010; 79: 141-6.

21. Monajemzadeh M, Mokhtari S, Motamed F, et al. Plasma ghrelin levels in children with cystic fibrosis and healthy children. Arch Med Sci 2013; 9: 93-7.

22. Cohen RI, Ginsberg N, Tsang D, Wann LC, Ye X, Liu SF. Association of nesfatin-1 and fat mass in cystic fibrosis. Respir Int Rev Thorac Dis 2013; 86: 312-7.

23. Guo Y, Su M, McNutt MA, Gu J. Expression and distribution of cystic fibrosis transmembrane conductance regulator in neurons of the human brain. J Histochem Cytochem 2009; 57: 1113-20.

24. Xue R, Gu H, Qiu Y, et al. Expression of cystic fibrosis transmembrane conductance regulator in ganglia of human gastrointestinal tract. Sci Rep 2016; 6: 30926.

25. Varness T, Seffrood EE, Connor EL, Rock MJ, Allen DB. Oxandrolone improves height velocity and $\mathrm{BMI}$ in patients with cystic fibrosis. Int J Pediatr Endocrinol 2009; 2009: 826895.

26. Yoshida T. Serum levels of mature brain-derived neurotrophic factor (BDNF) and its precursor proBDNF in healthy subjects. Open Clin Chem J 2012; 5: 7-12.

27. Russo F, Chimienti G, Clemente C, Ferreri C, Orlando A, Riezzo G. A possible role for ghrelin, leptin, brain-derived neurotrophic factor and docosahexaenoic acid in reducing the quality of life of coeliac disease patients following a gluten-free diet. Eur J Nutr 2017; 56: 807-18. 28. Liu W, Han X, Zhou X, et al. Brain derived neurotrophic factor in newly diagnosed diabetes and prediabetes. Mol Cell Endocrinol 2016; 429: 106-13.

29. Monteleone P, Fabrazzo M, Martiadis V, Serritella C, Pannuto M, Maj M. Circulating brain-derived neurotrophic factor is decreased in women with anorexia and bulimia nervosa but not in women with binge-eating disorder: relationships to co-morbid depression, psychopathology and hormonal variables. Psychol Med 2005; 35: 897-905.

30. Chimienti G, Mezzapesa A, Rotelli MT, Lupo L, Pepe G. Plasma concentrations but not serum concentrations of brain-derived neurotrophic factor are related to pro-in- 
flammatory cytokines in patients undergoing major abdominal surgery. Clin Biochem 2012; 45: 631-6.

31. Kauer-Sant'Anna M, Kapczinski F, Andreazza AC, et al. Brain-derived neurotrophic factor and inflammatory markers in patients with early- vs. late-stage bipolar disorder. Int J Neuropsychopharmacol 2009; 12: 447-58.

32. Huang T, Gejl AK, Tarp J, Andersen LB, Peijs L, Bugge A. Cross-sectional associations of objectively measured physical activity with brain-derived neurotrophic factor in adolescents. Physiol Behav 2017; 171: 87-91.

33. Anusha K, Hettiaratchi UPK, Athiththan LV, Perera PPR. Inter-relationship of serum leptin levels with selected anthropometric parameters among a non-diabetic population: a cross-sectional study. Eat Weight Disord 2019; 24 :551-556.

34. Wang D, Gao L, Gong K, Chai Q, Wang G. Increased serum leptin level in overweight patients with colon carcinoma: a cross-sectional and prospective study. Mol Clin Oncol 2017; 6: 75-8.

35. Domínguez C, Vieites-Prado A, Pérez-Mato $M$, et al. Role of adipocytokines in the pathophysiology of migraine. A cross-sectional study. Cephalalgia 2018; 38: 904-11.

36. Huang Q, Liao J, Liu Y, Liang H, Ma P, Pan J. Plasma neuropeptide $Y$ levels in Chinese patients with primary insomnia. Sleep Breath 2015; 19: 617-22.

37. Doniec Z, Pierzchała-Koziec K, Tomalak W, Kurzawa R. Serum level of leptin and neuropeptide $Y$ in children with mild asthma [Polish]. Pneumonol Alergol Pol 2004; 72: 9-13.

38. Vats P, Singh SN, Singh VK, et al. Appetite regulatory peptides in Indian Antarctic expeditioners. Nutr Neurosci 2005; 8: 233-8.

39. Mpouzika MDA, Papathanassoglou EDE, Giannakopoulou $\mathrm{M}$, et al. Altered serum stress neuropeptide levels in critically ill individuals and associations with lymphocyte populations. Neuropeptides 2013; 47: 25-36. 